\title{
Mesenteric tissue oxygenation status on the development of necrotizing enterocolitis
}

\author{
Hilal Özkan ${ }^{1 \oplus}$, Merih Çetinkaya ${ }^{2 \oplus}$, Bayram Ali Dorum $^{3 \oplus}$, Nilgün Köksal ${ }^{1 \oplus}$ \\ ${ }^{1}$ Department of Neonatology, Uludă̆ University Faculty of Medicine, Bursa; ${ }^{2}$ Department of Neonatology, Başakşehir Çam and Sakura \\ City Hospital, İstanbul; ${ }^{3}$ Department of Neonatology, Bursa City Hospital, Bursa, Turkey.
}

\begin{abstract}
Background. Necrotizing enterocolitis (NEC) is an important cause of morbidity and mortality in preterm infants. There is limited data about the role of mesenteric oxygenation status during the first enteral feeding. Therefore, the aim of this study was to determine the mesenteric tissue oxygen saturation values before, during and after the first enteral feeding and to evaluate the effect of these values on the development of NEC in preterm infants.

Methods. A total of 105 preterm babies with $\leq 32$ gestational weeks were included in this prospective study. The continuous monitoring of the mesenteric tissue oxygenation status was performed before, during and 3 hours after the first feeding by near-infrared spectroscopy (NIRS).

Results. The mean gestational week and birth weight of the study group were $28.8 \pm 2.1$ weeks, and $1215 \pm 387 \mathrm{~g}$, respectively. The first enteral feeding was started at $2.4 \pm 1.4$ days with breast milk in $85 \%$ of infants. A total of 12 infants (11.4\%) developed NEC (66\% stage II, 34\% stage III). The mean mesenteric tissue oxygen saturation levels of the infants that developed NEC were significantly lower both before and one hour after feeding (56.1 \pm 3.4 vs. $34 \pm 8.8$, and $47.4 \pm 3.3$ vs $37.8 \pm 10.9$, respectively) compared with infants that did not develop NEC.

Conclusions. Lower mesenteric tissue oxygenation values measured before, and one hour after enteral feeding was associated with NEC development. We suggest that lower mesenteric tissue oxygenation during continuous monitoring of first enteral feeding may be used to predict NEC development during follow-up.
\end{abstract}

Key words: enteral feeding, near-infrared spectroscopy, necrotizing enterocolitis, NICU, premature.

Necrotizing enterocolitis (NEC) is the most frequent and lethal gastrointestinal tract emergency in preterm newborns. ${ }^{1}$ Though more than 50 years have passed since its definition, its pathophysiology has not been elucidated completely. ${ }^{1}$ Prematurity, bacterial colonization, formula feeding, and intestinal ischemia were reported as the main risk factors that contribute to the complex pathogenesis. ${ }^{2}$ The most accepted NEC hypothesis includes enteral

$凶$ Hilal Özkan

hiozkan@hotmail.com

Received 23rd November 2020, revised 12th March 2021, 1st April 2021, accepted 21st April 2021.

This study was presented as an oral presentation at the 25th National Neonatology Congress held in Antalya in April 2017. feeding in the presence of intestinal hypoxiaischemia-reperfusion, and abnormal intestinal colonization with pathogens that provoke an inappropriate inflammatory response in intestinal epithelial cells of premature infants. ${ }^{3,4}$

Therefore, it seems reasonable to establish the intestinal oxygenation status before and during the first enteral feeding attempts in premature infants and determine the high-risk infants for the development of NEC.

Near-infrared spectroscopy (NIRS) has been increasingly used to provide continuous monitoring of tissue oxygen saturation $\left(\mathrm{StO}_{2}\right)$ in neonates, especially for cerebral, renal and mesenteric oxygenation. ${ }^{5}$ Limited number of studies investigated the effect of intestinal oxygenation status during enteral feeding as a 
biomarker of mesenteric perfusion on feeding intolerance and intestinal complications in preterm infants. ${ }^{6-9}$ In addition, lower mesenteric tissue oxygenation and increased fractional tissue oxygen extraction (FTOE) was found in preterm infants during the early course of NEC. ${ }^{10}$ Also, low mesenteric/cerebral oxygen saturation values and high mesenteric/cerebral FTOE levels were reported to be associated with bowel perforation or death in premature infants with NEC. ${ }^{11}$

However, to our best of knowledge, no study evaluated the possible association between mesenteric tissue oxygenation during the first enteral feeding and subsequent development of NEC in preterm infants. The aim of this prospective study was to determine the continuous mesenteric tissue oxygenation during the first enteral feeding and also to explore the possible association between these values and subsequent NEC development during the follow-up period.

\section{Material and Methods}

\section{Study population}

This prospective observational study was performed at two tertiary Neonatal Intensive Care Units. Preterm infants $\leq 32$ gestational weeks, and hospitalized in these two centers between December 2015 and December 2017 were included. Infants with gastrointestinal/major congenital and/ or genetic anomalies, hemodynamically significant patent ductus arteriosus (diameter $\geq 2.0 \mathrm{~mm}$ or left atrium to aortic root ratio $\geq 1.4$ or retrograde flow in descending aorta), severe intraventricular hemorrhage (grade III, IV) and hemodynamically unstable patients, need of volume or inotrope treatment, babies who have anemia (hemoglobin $<12 \mathrm{~g} / \mathrm{dl}$ ) and need red blood cell transfusion, infants died within the first 3 days of life were all excluded.

Nasal continuous positive airway pressure (NCPAP) $\left(6 \mathrm{cmH}_{2} \mathrm{O}\right)$ was applied to all babies after birth. They were transferred to the neonatal intensive care unit (NICU) with nasal CPAP. In nasal CPAP failure, firstly non-synchronized nasal intermittent positive pressure ventilation was applied. However, babies whose respiratory support was insufficient were intubated and received ventilation support in volumeguaranteed mode. Target oxygen saturation was targeted between 90-94\%. Intratracheal surfactant were administered to infants who needed $\mathrm{FiO}_{2}$ more than 0.30 for target saturation.

Uludag University Faculty of Medicine Clinical Research Ethics Committee approved the study (2012-26/11) and the signed approved parental consent was obtained from all families.

\section{Study design}

All of the infants were started total parenteral nutrition during the admission and enteral feeding was started with breast milk as soon as possible and if not available with preterm formula at amounts of $10-20 \mathrm{ml} / \mathrm{kg}$ given at 3-hour intervals as a bolus. With the decision of enteral feeding, mesenteric, renal, and cerebral $\mathrm{StO}_{2}$ were measured by NIRS for a 4 hours period starting from 1 hour before the first feeding, and continued during 3 hours of enteral feeding. The systemic oxygen saturation $\left(\mathrm{SaO}_{2}\right)$ of the infants were monitored simultaneously using a pulse oximetry device (Nellcor, CovidienMedtronic, Minneapolis, US). The patients were followed up for the development of NEC. During follow-up, modified Bell criteria were used for the diagnosis, and staging of infants with NEC. ${ }^{12}$ Demographical, prenatal and natal characteristics, and neonatal morbidities were all recorded. The infants with stage I NEC were excluded.

\section{Near-infrared spectroscopy}

INVOS 5100 near-infrared spectroscopy (Covidien, Mansfield, US) was used. NIRS data were recorded at 6-second intervals. Cerebral and renal sensors were placed on the anterior frontal region and on the left lumbar region, respectively. For mesenteric $\mathrm{StO}_{2}$ measurement, sensors were placed on the infraumbilical region at the center of the abdominal wall. 
Mean cerebral, renal, and mesenteric NIRS data obtained 1 hour before, during and 1, 2, and 3 hours after first enteral feeding were calculated. To minimize erroneous measurements stemming from movements, and malposition of the sensors, mean values of all measurements performed within +/- 15 minutes were taken into consideration. FTOE of the patients were calculated using the following formula: $\mathrm{FTOE}=\mathrm{SaO}_{2}-\mathrm{StO}_{2} / \mathrm{SaO}_{2}$.

\section{Statistical analysis}

Data were analyzed using the IBM Statistical Package for Social Sciences v23 (SPSS Inc., Chicago, IL, USA). Continuous data were presented as mean \pm standard deviation or median (minimum-maximum), as appropriate. All differences associated with a chance probability of 0.05 or less were considered statistically significant. Receiver-operating characteristics (ROC) analysis was performed by MedCalc version 18.2.1 statistical program. Values of $\mathrm{p}<0.05$ were considered significant.

\section{Results}

During a period of two years, a total of 147 babies born at a gestational age of $\leq 32$ weeks were hospitalized in the NICU. When the infants with exclusion criteria and without parental consent were excluded, 105 preterm babies were included in the study (Fig. 1).

The mean gestational age, and birth weights of the study group were $28.8 \pm 2.1$ weeks, and $1215 \pm 387 \mathrm{~g}$, respectively. The mean first enteral feeding time was $2.4 \pm 1.4$ days and $85 \%$ of the patients were breastfed. A total of 12 infants $(11,4 \%)$ developed NEC; $8(7,6 \%)$ had stage 2 and $4(3.8 \%)$ had stage 3 . Although there were no significant differences in infants with and without NEC in terms of demographical features, the time of first enteral feeding was statistically significantly later in babies with NEC $(p<0.001)$ (Table I).

No significant differences were detected between infants with and without NEC in terms of mean cerebral and renal $\mathrm{StO}_{2}$. The mean mesenteric $\mathrm{StO}_{2}$ were significantly lower before feeding $(56.1 \pm 3.4$ vs. $34 \pm 8.8)$ and one hour after feeding $(47.4 \pm 3.3$ vs $37.8 \pm 10.9)$ in cases that subsequently developed NEC compared with those who did not develop NEC (Table II). In the NEC group, mesenteric tissue oxygen saturations at the 2 nd and 3rd hours after the first enteral feeding were found to be lower, but this difference was not significant (Table II). Similarly, FTOE levels before and 1 hour after feeding were significantly higher in cases that developed NEC (Fig. 2). The ROC analysis showed a cut-off of $42,43,47,45 \%$ for before the first feeding, the first hour after first feeding, the second hour after first feeding and, the third hour after the first feeding respectively for prediction of NEC (Table III).

\section{Discussion}

To the best of our knowledge, this is the first study that evaluated the possible role

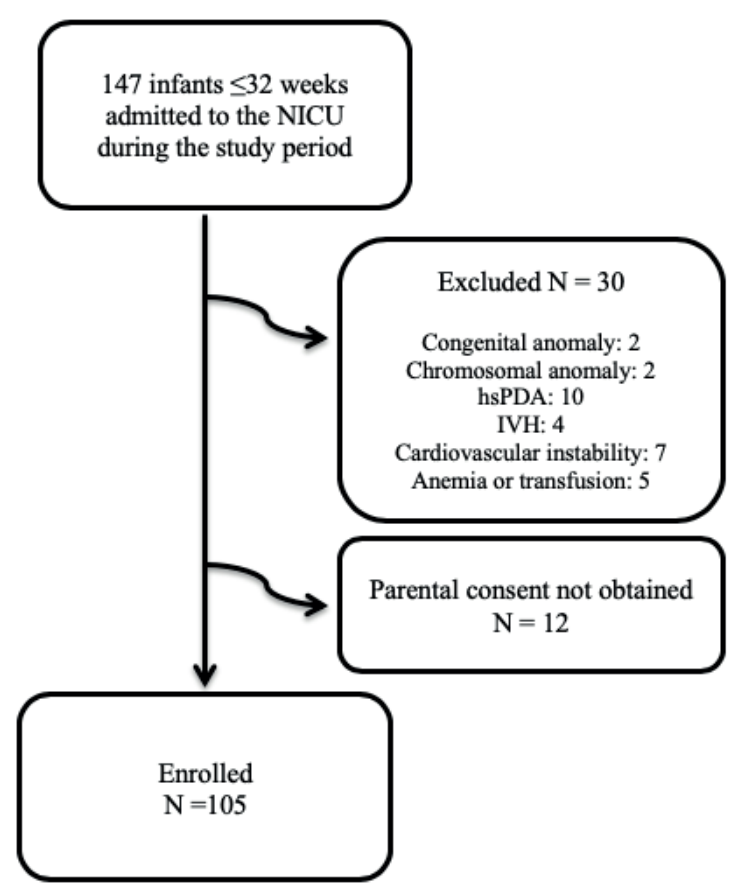

Fig. 1. Flow chart of the study enrollment (NICU: Neonatal intensive care unit, hsPDA: Hemodynamically Significant Patent Ductus Arteriosus, IVH: Intraventricular hemorrhage). 
Table I. Demographics and characteristics of all infants enrolled in the study.

\begin{tabular}{lcc}
\hline & NEC group n=12 & Non-NEC group n=93 \\
\hline Maternal features & $7(58.3)$ & $35(37.6)$ \\
Preeclampsia, n (\%) & $1(8.3)$ & $7(7.5)$ \\
Premature rupture of membranes, n (\%) & $96(70.9)$ \\
Antenatal steroid, $\mathrm{n}(\%)$ & $10(83.3)$ & $77(82.7)$ \\
Caesarian section, $\mathrm{n}(\%)$ & & $1237 \pm 358$ \\
Neonatal features & $1068 \pm 335$ & $28.9 \pm 2.2$ \\
Birth weight $(\mathrm{g})$, mean \pm std & $28.1 \pm 1.3$ & $20(21.5)$ \\
Gestational age, mean \pm std & $4(33.3)$ & $46(49.4)$ \\
Small gestational age (<10 percentile), $\mathrm{n}(\%)$ & $7(58.3)$ & $4.8 \pm 2.1$ \\
Male gender, $\mathrm{n}(\%)$ & $5.7 \pm 2.0$ & $6.8 \pm 1.7$ \\
Apgar score-1, mean \pm std & $7.0 \pm 1.7$ & $60(64)$ \\
Apgar score-5, mean \pm std & $9(75)$ & $50(53.7)$ \\
Respiratory distress syndrome, $\mathrm{n}(\%)$ & $8(66.6)$ & $80(86)$ \\
Mechanical ventilation, $\mathrm{n}(\%)$ & $10(83.3)$ & $2.2 \pm 0.9$ \\
Human milk feeding, $\mathrm{n}(\%)$ & $4.0 \pm 2.6$ & - \\
First day of enteral feeding, mean \pm std ${ }^{*}$ & $12.3 \pm 5.3$ & $20(21.5)$ \\
Development of NEC, day & $4(33.3)$ & $5.7 \pm 3.3$ \\
Probiotic supplementation, $\mathrm{n}(\%)$ & $7.5 \pm 2.6$ & \\
CRIB-II Score, mean \pm std & & \\
\hline
\end{tabular}
${ }^{*} \mathrm{p}=0.0001$

Table II. Cerebral, renal, and mesenteric tissue oxygen saturation $\left(\mathrm{StO}_{2}\right)$ values of infants before and after first feeding.

\begin{tabular}{|c|c|c|}
\hline & NEC group $n=12$ & Non-NEC group $n=93$ \\
\hline \multicolumn{3}{|l|}{ Before first feeding (\%) } \\
\hline - Cerebral $\mathrm{StO}_{2^{\prime}}$ mean \pm std & $69 \pm 7.3$ & $70 \pm 13.2$ \\
\hline - Renal $\mathrm{StO}_{2}$, mean \pm std & $64.6 \pm 17.2$ & $66.2 \pm 20$ \\
\hline - Mesenteric $\mathrm{StO}_{2^{\prime}}$ mean $\pm \mathrm{std}^{*}$ & $34.8 \pm 10.9$ & $56.1 \pm 3.4$ \\
\hline \multicolumn{3}{|l|}{ 1st hour after feeding (\%) } \\
\hline - Cerebral StO ${ }_{2}$, mean \pm std & $64.5 \pm 8.3$ & $69.7 \pm 13$ \\
\hline - Renal $\mathrm{StO}_{2}$, mean \pm std & $66.1 \pm 16.4$ & $68 \pm 19.2$ \\
\hline - Mesenteric $\mathrm{StO}_{2}$, mean $\pm \mathrm{std}^{*}$ & $37.8 \pm 10.9$ & $47.4 \pm 3.3$ \\
\hline \multicolumn{3}{|l|}{ 2nd hour after feeding (\%) } \\
\hline - Cerebral $\mathrm{StO}_{2}$, mean \pm std & $69 \pm 7.8$ & $68.1 \pm 15.8$ \\
\hline - Renal $\mathrm{StO}_{2}$, mean \pm std & $72 \pm 18.8$ & $70 \pm 18.7$ \\
\hline - Mesenteric $\mathrm{StO}_{2}$, mean \pm std & $40 \pm 23$ & $52 \pm 24$ \\
\hline \multicolumn{3}{|l|}{ 3rd hour after feeding (\%) } \\
\hline - Cerebral $\mathrm{StO}_{2^{\prime}}$ mean \pm std & $67 \pm 8.1$ & $67.8 \pm 17.4$ \\
\hline - Renal $\mathrm{StO}_{2}$, mean \pm std & $71 \pm 17.5$ & $65.4 \pm 20.5$ \\
\hline - Mesenteric $\mathrm{StO}_{2^{\prime}}$ mean \pm std & $41.7 \pm 23$ & $49.5 \pm 25$ \\
\hline
\end{tabular}

NEC: necrotizing enterocolitis, $\mathrm{StO}_{2}$ : tissue oxygen saturation ${ }^{*} \mathrm{p}=0.0001$ 


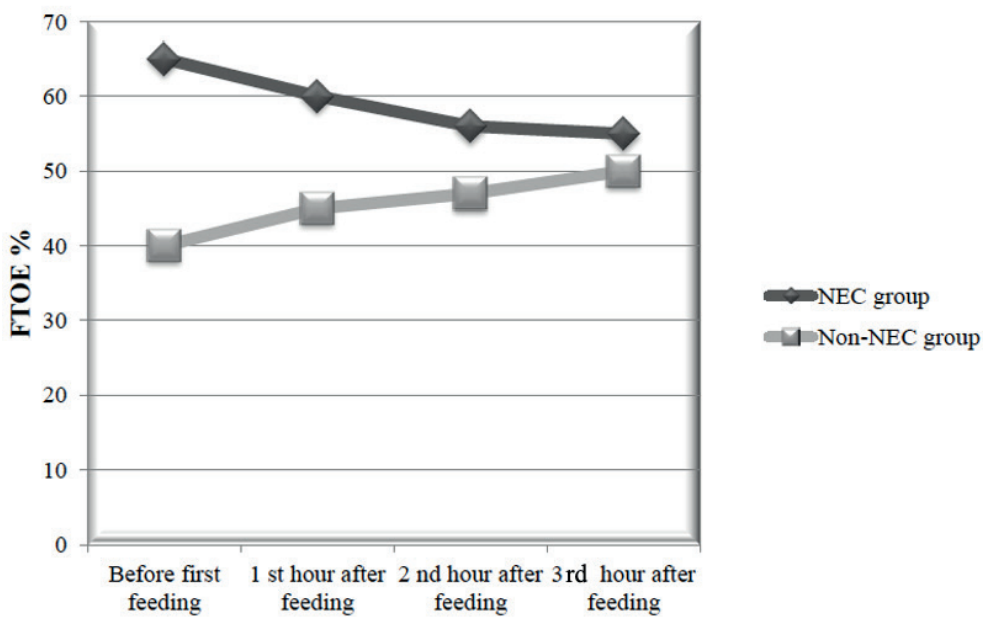

Fig. 2. Fractional tissue oxygen extraction (FTOE) values of infants with necrotizing enterocolitis (NEC) and non-NEC infants before and after first feeding.

Table III. Criterion values and coordinates of the ROC curve.

\begin{tabular}{lcccccc}
\hline $\begin{array}{l}\text { Variable } \\
\text { Mesenteric } \mathrm{StO}_{2^{\prime}}(\%)\end{array}$ & Cut off & Sensitivity & Specificity & AUC & 95\% Cl & $\mathrm{P}$ \\
\hline Before first feeding & 42 & 83 & 100 & 0.987 & 0.890 to 1.000 & $<0.001$ \\
1st hour after feeding & 43 & 83 & 92 & 0.924 & 0.797 to 0.983 & $<0.001$ \\
2nd hour after feeding & 47 & 75 & 93 & 0.813 & 0.661 to 0.918 & $<0.001$ \\
3rd hour after feeding & 45 & 83 & 87 & 0.833 & 0.684 to 0.931 & 0.005 \\
\hline
\end{tabular}

ROC: receiver operating characteristic, $\mathrm{StO}_{2}$ : tissue oxygen saturation, $\mathrm{AUC}$ : area under the ROC curve

of mesenteric tissue oxygenation during the introduction of first enteral feeding and subsequent development of NEC in preterm infants. This study suggested that lower mesenteric $\mathrm{StO}_{2}$ and higher FTOE values before, and during the first hour of the enteral feeding were associated with the development of NEC during the follow-up period.

The incidence of NEC in very-low birth weight (VLBW) babies was reported up to $13 \%$ according to the large multicenter studies and neonatal networks. ${ }^{13}$ The incidence of severe NEC $(11.4 \%)$ in our study was similar to the literature. Despite major recent developments in the care of preterm infants, a highly sensitive and specific test for the early diagnosis of NEC is still lacking.

As prematurity, ischemia, feeding and abnormal colonization were reported as the main risk factors, a non-invasive diagnostic approach for these risk factors may provide early and accurate NEC diagnosis in preterm infants. NIRS has been increasingly used in the last years for the assessment of cerebral perfusion in both term and preterm infants. In addition, the non-invasive measurement of $\mathrm{StO}_{2}$ and FTOE may be also calculated by the $\mathrm{SaO}_{2}$ measurements. ${ }^{11}$

As the ischemic necrosis of intestinal mucosa is a stable sign in the histopathological examination of advanced stage NEC, detection of the decrease in the abdominal $\mathrm{StO}_{2}$ related to mesenteric perfusion alterations before the development of NEC may offer a very reasonable diagnostic approach. Indeed, animal studies also yielded that lower NIRS measurements might be used for the early diagnosis of NEC. ${ }^{14}$

After these experimental data, abdominal $\mathrm{StO}_{2}$ were found to be significantly lower in infants that developed NEC in a two-centered clinical 
study and abdominal $\mathrm{StO}_{2}<56 \%$ were stated as an independent risk factor for the development of NEC in preterm infants. ${ }^{15}$ The authors also reported significantly more variations both during and after feeding in the first two weeks of life. Our results were in accordance with this study as the mean mesenteric saturation levels were always lower than 56\% in infants before, during and after first enteral feeding in premature infants. Therefore, we may speculate that lower mesenteric oxygen saturation, especially lower than $42 \%$ may predict subsequent NEC development during the hospitalization period.

A strong association between mesenteric FTOE and intestinal fatty acid binding protein levels were reported during the first 16 hours after NEC onset that suggested the simultaneous occurrence of decreased splanchnic perfusion and intestinal damage. ${ }^{10}$ The authors suggested that mesenteric FTOE might offer valuable information about the degree of intestinal injury. NIRS monitoring was reported to be useful in preterm infants with definite NEC to differentiate the infants who would develop complicated NEC. ${ }^{11}$ The lower mesenteric and cerebral oxygenation values and increased FTOE were also found to be associated with adverse outcomes including bowel perforation and death. ${ }^{11}$ Similarly, increased mesenteric FTOE during the first enteral feeding was detected in our study. Therefore, we suggest that increased mesenteric FTOE levels may help neonatologists to identify the high-risk infants for NEC development. However, we could not find any differences in both cerebral and renal oxygenation levels in association with first enteral feedings to predict subsequent NEC development.

There are conflicting data about the correlation between mesenteric tissue oxygenation and feeding intolerance during the first introduction of enteral feeding in preterm infants. In a clinical study, lower abdominal saturations and mesenteric-cerebral oxygenation ratio were detected in infants that developed feeding intolerance. ${ }^{7}$ Similarly, lower mesenteric oxygenation and increased FTOE were reported in response to both initial and full enteral feedings in infants with absent/reversed antenatal end diastolic flow (AREDF). ${ }^{12}$ As both feeding intolerance and AREDF are important risk factors for NEC, these results should be interpreted in this manner. In contrast, abdominal $\mathrm{StO}_{2}$ recorded during the first postnatal days was found to not provide helpful information about nutritional tolerance in the follow-up period. ${ }^{6}$ This difference may be explained by the cerebral autoregulation mechanisms that keep tissue oxygenation stable.

Although the superior mesenteric artery flow rates after feeding show an increase in healthy preterm infants, this finding was not detected by Doppler US in infants who later developed feeding intolerance or NEC. ${ }^{16}$ Contrary to Doppler US, NIRS provides continuous data about mesenteric oxygenation without the need of trained personnel. In our study, persistently lower mesenteric oxygenation levels were detected in infants that subsequently developed NEC. Therefore, we may suggest using NIRS alone or in combination with Doppler US for the prediction of NEC earlier in high-risk preterm infants.

In conclusion, NIRS may provide valuable data about the intestinal oxygenation status during the first enteral feeding in preterm infants. Lower mesenteric $\mathrm{StO}_{2}$ and increased FTOE levels in preterm infants with normal cerebral and renal $\mathrm{StO}_{2}$ during the introduction of first enteral feeding may predict subsequent NEC development. NIRS findings may be used to determine, characteristics of enteral feeding in high-risk infants for NEC development. However, prospective studies including a larger number of infants with prolonged NIRS monitorization periods are required to elucidate the exact role of intestinal tissue oxygenation during the early enteral feeding on subsequent NEC development in preterm infants. 


\section{Acknowledgments}

The project was supported by the funds from scientific research project council of our university (ID: OUAP(T) - 2013/24).

\section{Author contribution}

The contributions of all authors must be described in the following manner: The authors confirm contribution to the paper as follows: study conception and design: $\mathrm{HO}, \mathrm{MÇ}$, NK; data collection: BAD, MÇ; analysis and interpretation of results: $\mathrm{HO}, \mathrm{MC}, \mathrm{NK}$; draft manuscript preparation: BAD, HO. All authors reviewed the results and approved the final version of the manuscript

\section{Ethical approval}

Uludag University Faculty of Medicine Clinical Research Ethics Committee approved the study (2012-26/11) and the signed approved parental consent was obtained from all families.

\section{Conflict of interest}

Authors state no conflict of interest.

\section{REFERENCES}

1. Schat TE, van der Laan ME, Schurink $M$, et al. Abdominal near-infrared spectroscopy in preterm infants: a comparison of splanchnic oxygen saturation measurements at two abdominal locations. Early Hum Dev 2014; 90: 371-375.

2. Frost BL, Modi BP, Jaksic T, Caplan MS. New medical and surgical insights into neonatal necrotizing enterocolitis: a review. JAMA Pediatr 2017; 171: 8388 .

3. Wu SF, Caplan M, Lin HC. Necrotizing enterocolitis: old problem with new hope. Pediatr Neonatol 2012; 53: 158-163.

4. Chen AC, Chung MY, ChangJH, Lin HC.Pathogenesis implication for necrotizing enterocolitis prevention in preterm very-low-birth-weight infants. J Pediatr Gastroenterol Nutr 2014; 58: 7-11.
5. Bozzetti V, Paterlini G, Bel FV, et al. Cerebral and somatic NIRS- determined oxygenation in IUGR preterm infants during transition. J Matern Fetal Neonatal Med 2016; 29: 443-446.

6. Dani C, Corsini I, Generoso M, Gozzini E, Bianconi T, Pratesi S. Splanchnic tissue oxygenation for predicting feeding tolerance in preterm infants. JPEN J Parenter Enter Nutr 2015; 39: 935-940.

7. Corvaglia L, Martini S, Battistini B, Rucci P, Faldella G, Aceti A. Splanchnic oxygenation at first enteral feeding in preterm infants: correlation with feeding intolerance. J Pediatr Gastroenterol Nutr 2017; 64: 550-554.

8. Martini S, Aceti A, Beghetti I, Faldella G, Corvaglia L. Feed-related splanchnic oxygenation in preterm infants with abnormal antenatal Doppler developing gut complications. J Pediatr Gastroenterol Nutr 2018; 66: 755-759.

9. Cortez J, Gupta M, Araram A, Pizzino J, Sawhney M, Sood BG. Noninvasive evaluation of splanchnic tissue oxygenation using near-infrared spectroscopy in preterm infants. J Matern Fetal Neonatal Med 2011; 24: 578-582.

10. Schat TE, Heida FH, Schurink, et al. The relation between splanchic ischemia and intestinal damage in necrotizing enterocolitis. Arch Dis Child Fetal Neonatal Ed 2016; 101: F533-F539.

11. Schat TE, Schurink M, van der Laan ME, et al Near-infrared spectroscopy to predict the course of necrotizing enterocolitis. PLoS One 2016; 11: e0154710.

12. Walsh MC, Kliegman RM. Necrotizing enterocolitis: treatment based on staging criteria. Pediatr Clin North Am 1986; 33: 179-201.

13. Nino DF, Sodhi CP, Hackam DJ. Necrotizing enterocolitis: new insights into pathogenesis and mechanisms. Nat Rev Gastroenterol Hepatol 2016; 13: 590-600.

14. Gay AN, Lazar DA, Stoll B, et al. Near infrared spectroscopy measurement of abdominal tissue oxygenation is a useful indicator of intestinal blood flow and necrotizing enterocolitis in premature piglets. J Pediatr Surg 2011; 46: 1034-1040.

15. Patel AK, Lazar DA, Burrin DG, et al. Abdominal near-infrared spectroscopy measurements are lower in preterm infants at risk for necrotizing enterocolitis. Pediatr Crit Care Med 2014; 15: 735-741.

16. Bora R, Mukhopadhyay K, Saxena AK, Jain V, Narang A. Prediction of feed intolerance and necrotizing enterocolitis in neonates with absent end diastolic flow in umbilical artery and the correlation of feed intolerance with postnatal superior mesenteric artery flow. J Matern Neonatal Med 2009; 22: 10921096. 